\title{
A noninvasive estimation of cerebral perfusion pressure using critical closing pressure
}

\author{
Georgios V. Varsos, MSc, ${ }^{1}$ Angelos G. Kolias, MSc, MRCS, ${ }^{1}$ Peter Smielewski, PhD, ${ }^{1}$ \\ Ken M. Brady, MD, ${ }^{2}$ Vassilis G. Varsos, MD, PhD, ${ }^{3}$ Peter J. Hutchinson, PhD, ${ }^{1}$ \\ John D. Pickard, FMedSci, ${ }^{1}$ and Marek Czosnyka, PhD ${ }^{1,4}$
}

\begin{abstract}
'Division of Neurosurgery, Department of Clinical Neurosciences, Addenbrooke's Hospital, University of Cambridge, United Kingdom; 'Baylor College of Medicine, Texas Children's Hospital, Houston, Texas; ${ }^{3}$ Department of Neurosurgery, Red Cross Hospital, Athens, Greece; ${ }^{4}$ Institute of Electronic Systems, Warsaw University of Technology, Warsaw, Poland
\end{abstract}

\begin{abstract}
OBJECT Cerebral blood flow is associated with cerebral perfusion pressure (CPP), which is clinically monitored through arterial blood pressure (ABP) and invasive measurements of intracranial pressure (ICP). Based on critical closing pressure ( $\mathrm{CrCP})$, the authors introduce a novel method for a noninvasive estimator of CPP (eCPP).

METHODS Data from 280 head-injured patients with ABP, ICP, and transcranial Doppler ultrasonography measurements were retrospectively examined. $\mathrm{CrCP}$ was calculated with a noninvasive version of the cerebrovascular impedance method. The eCPP was refined with a predictive regression model of CrCP-based estimation of ICP from known ICP using data from 232 patients, and validated with data from the remaining 48 patients.

RESULTS Cohort analysis showed eCPP to be correlated with measured CPP $(R=0.851, p<0.001)$, with a mean $\pm S D$ difference of $4.02 \pm 6.01 \mathrm{~mm} \mathrm{Hg}$, and $83.3 \%$ of the cases with an estimation error below $10 \mathrm{~mm} \mathrm{Hg}$. eCPP accurately predicted low $\mathrm{CPP}(<70 \mathrm{~mm} \mathrm{Hg})$ with an area under the curve of $0.913(95 \% \mathrm{Cl} 0.883-0.944)$. When each recording session of a patient was assessed individually, eCPP could predict CPP with a $95 \% \mathrm{Cl}$ of the SD for estimating CPP between multiple recording sessions of $1.89-5.01 \mathrm{~mm} \mathrm{Hg}$.
\end{abstract}

CONCLUSIONS Overall, CrCP-based eCPP was strongly correlated with invasive CPP, with sensitivity and specificity for detection of low CPP that show promise for clinical use.

http://thejns.org/doi/abs/10.3171/2014.10.JNS14613

KEY WORDS cerebral perfusion pressure; critical closing pressure; noninvasive model; transcranial Doppler ultrasonography; vascular disorders

$\mathrm{T}$ HE circulation of cerebral blood flow (CBF) is driven by cerebral perfusion pressure (CPP), which is defined as the vascular pressure gradient across the cerebral bed and can be calculated as the difference between arterial blood pressure (ABP) and pressure in cor- tical or bridging veins. ${ }^{15,24}$ Due to difficulties in measuring the pressure of bridging veins, invasive intracranial pressure (ICP) measurements are used instead as an approximation, defining CPP as ABP - ICP. ${ }^{1,26,34,39}$ Cerebral perfusion pressure in clinical practice is considered an es-

\footnotetext{
ABBREVIATIONS $\mathrm{ABP}=$ arterial blood pressure; $\mathrm{AUC}=$ area under the curve; $\mathrm{CBF}=$ cerebral blood flow; $\mathrm{CPP}=$ cerebral perfusion pressure; $\mathrm{CrCP}=$ critical closing pressure; $\mathrm{CVR}$ = cerebrovascular resistance; $\mathrm{eCPP}=$ noninvasive estimator of CPP; FV = flow velocity; GCS = Glasgow Coma Scale; GOS = Glasgow Outcome Scale; ICP = intracranial pressure; IQR = interquartile range; $M C A=$ middle cerebral artery; nICP = noninvasive estimator of ICP; ROC = receiver operating characteristic; TAU = time constant of the cerebrovascular arterial bed; $\mathrm{TBI}=$ traumatic brain injury; $\mathrm{TCD}=$ transcranial Doppler.

SUBMITTED March 17, 2014. ACCEPTED October 21, 2014.

INCLUDE WHEN CITING Published online January 9, 2015; DOI: 10.3171/2014.10.JNS14613.

DISCLOSURE G. Varsos is supported by an A. G. Leventis Foundation Scholarship and a Charter Studentship from St. Edmund's College, Cambridge. Dr. Kolias is supported by a Royal College of Surgeons of England Research Fellowship, a National Institute for Health Research (NIHR) Academic Clinical Fellowship, and a Raymond and Beverly Sackler Studentship. He also chairs the British Neurosurgical Trainee Research Collaborative, which has been supported with an educational grant from Codman. Dr. Hutchinson is supported by an NIHR Research Professorship, the NIHR Cambridge Biomedical Research Centre, and has been appointed as the Surgical Specialty Lead for Neurosurgery, Royal College of Surgeons of England Clinical Research Initiative. He is a director of Technicam, a manufacturer of cranial access devices for neuromonitoring. He has also received honoraria from Codman. J. Pickard's research (excluding salary) is supported by the NIHR Cambridge Biomedical Research Centre and an NIHR Senior Investigator Award. ICM+ Software is licensed by Cambridge Enterprise, Cambridge, UK, and Dr. Czosnyka and Dr. Smielewski have a financial interest in a fraction of the licensing fee. Dr. Czosnyka has also served as a consultant to Codman.
} 
sential monitored parameter for head-injury management protocols, ${ }^{7,9,12-14,22,23,31,33,35}$ even though ideal CPP has been poorly delineated., ${ }^{4,41}$ The invasive part of CPP calculation is ICP, the invasive nature of which can make the calculation of CPP and thus the CPP-oriented management unfeasible, such as in some clinical scenarios in which invasive measurement of ICP is either not available or unobtainable (i.e., for patients with contraindications for direct measurement). ${ }^{3,8,27}$ In contrast, ABP can be measured and monitored noninvasively, ${ }^{20,30,37}$ i.e., with a Finapres finger plethysmograph, the use of which has been shown to provide a good level of agreement between Finapres-derived indices of cerebral autoregulation and their corresponding estimates obtained from invasive measurements of aortic ABP. ${ }^{36}$

Cerebral perfusion pressure has been approximated noninvasively with various methodologies in the past, based on ABP and transcranial Doppler (TCD) ultrasonography approximations of $\mathrm{CBF}^{2,5,10,18,38}$ The use of these methodologies is promising as their estimation accuracy is improving. We have created a new method for assessing CPP noninvasively using a model based on the concept of critical closing pressure $(\mathrm{CrCP})$, requiring $\mathrm{ABP}$ and TCD flow velocity (FV) measurements.

Critical closing pressure denotes a threshold of ABP, below which the local microvascular blood pressure is inadequate to prevent collapse and cessation of blood flow. ${ }^{28}$ As CrCP is associated with the vasomotor tone of small blood vessels, knowledge of its behavior has been recognized as able to provide valuable information regarding the state of cerebral hemodynamics in different pathologies. ${ }^{11,25,28,32,40}$ We have recently introduced a new method for estimating $\mathrm{CrCP}$ that is an apparent improvement on traditional methodology, ${ }^{25,40}$ in which the new, impedance model-based method does not render negative values. ${ }^{32,40}$ However, this method is invasive, as ICP measurements are required, possibly limiting its clinical applications in situations in which measuring ICP is not an option. For this reason, an equivalent noninvasive version of this model has been introduced, which only requires measurements of $\mathrm{ABP}$ and $\mathrm{FV}$. The primary aim of this study was to use the CrCP method for a noninvasive assessment of CPP in a large group of head-injured patients.

\section{Methods \\ Patient Population}

The presented analysis was performed as part of an anonymous clinical audit, with approval of the Neurocritical Care Users Committee of Addenbrooke's Hospital in Cambridge, the United Kingdom. This retrospective study included prospectively collected data from 280 sedated and ventilated patients with head injuries $(78.2 \%$ male, median age 29 years, interquartile range [IQR] 20-43 years), hospitalized in the Neurocritical Care Unit of Addenbrooke's Hospital between 2002 and 2011. ${ }^{10}$ The demographics of the patients are summarized in Table 1. All patients suffered a traumatic brain injury (TBI) and had an abnormal CT scan of the head. Patients were sedated, ventilated, and managed in the Neurocritical Care Unit with a tiered therapeutic protocol aiming for an ICP $<25 \mathrm{~mm} \mathrm{Hg}$ and CPP around $60-70 \mathrm{~mm} \mathrm{Hg}$. The median preintuba- tion Glasgow Coma Scale (GCS) score of the patients was 6 (range 3-15), while the Glasgow Outcome Scale (GOS) score, assessed 6 months after injury, varied from good outcome to death: 144 patients $(51.4 \%)$ had a favorable outcome (no disability to moderate disability), while 63 patients $(22.5 \%)$ died. Patients remaining in a persistent vegetative state $(n=9$, or $3.2 \%$ of the total number of patients) were excluded from the study. The data included daily recordings of $\mathrm{ABP}, \mathrm{ICP}$, and TCD, performed under a standard clinical brain monitoring protocol, in a total of 780 recording periods.

\section{Monitoring and Data Analysis}

To monitor ABP, a pressure monitoring kit (Baxter Healthcare) at the radial artery was used, zeroed at the level of the heart. Monitoring of ICP was performed via an intraparenchymal probe (Codman \& Shurtleff, or Camino Laboratories). Cerebral blood FV was measured from the middle cerebral artery (MCA) with a $2-\mathrm{MHz}$ probe and monitored with the Doppler Box (DWL Compumedics) or Neuroguard (Medasonics, Inc.). The TCD recordings were performed on a daily basis for periods ranging from 10 minutes up to 1 hour, starting from the day of initiation of invasive monitoring. Termination of the monitoring was decided based on clinical grounds. An analog-digital converter (DT2814 or DT9801, Data Translation) was used to digitize the raw data signals at a sampling frequency of $50 \mathrm{~Hz}$, which were then recorded using WREC (Warsaw University of Technology), BioSAn (University of Cambridge), or ICM+ (Cambridge Enterprise, http://www. neurosurg.cam.ac.uk/icmplus/) software. Heart rate was calculated using spectral position of the peak associated with the first harmonic of ABP. All calculations, including mean values of ABP, ICP, FV, and CPP, were performed over a sliding window of 10 seconds.

\section{Calculation of Noninvasive CrCP and CPP}

Critical closing pressure (expressed in $\mathrm{mm} \mathrm{Hg}$ ) was estimated through a noninvasive version of the impedance CrCP methodology, ${ }^{40}$ based on ABP and TCD FV (see Appendix):

$$
C r C P=A B P \cdot\left[1-\frac{1}{\sqrt{(C V R \cdot C a \cdot H R \cdot 2 \pi)^{2}+1}}\right]
$$

CVR denotes cerebrovascular resistance, Ca expresses arterial compliance of the cerebral bed, and HR represents heart rate (beats/sec).

To create and assess the model for the noninvasive estimator of CPP (eCPP), we dichotomized our set cohort of patients and formed 2 subgroups: the formation group with 232 patients (including 455 recordings) and the validation group with 48 patients (including 325 recordings).

Because the invasive part of CPP is ICP, we used the formation group to render a noninvasive estimator of ICP (nICP) based on a regression analysis of known ICP with $\mathrm{CrCP}$ (Eq. 1): $n I C P=0.266 \cdot C r C P+7.026(\mathrm{p}<0.001 ; \mathrm{R}$ $=0.340, \mathrm{~F}=59.225, \mathrm{~N}=455)$. Details of the regression analysis are presented in Table 2. 
TABLE 1. Patient characteristics

\begin{tabular}{|c|c|c|c|c|}
\hline Variable & Overall Cohort & Formation Group & Validation Group & $p$ Value* \\
\hline No. of patients & 280 & 232 & 48 & \\
\hline No. of recordings & 780 & 455 & 325 & \\
\hline \multicolumn{5}{|l|}{ Demographics } \\
\hline Median age in yrs (IQR) & $29(20-43)$ & $29(21-46)$ & $24(18-37)$ & 0.016 \\
\hline Males/females & 219:61 & $187: 45$ & $32: 16$ & \\
\hline Median GCS score before intubation (IQR) & $6(4-8)$ & $7(4-8)$ & $6(4-8)$ & 0.521 \\
\hline \multicolumn{5}{|l|}{ GOS score 6 mos after injury (\%) } \\
\hline Death & $63(22.5)$ & $54(23.3)$ & $9(18.8)$ & \\
\hline Persistent vegetative state $†$ & - & - & - & \\
\hline Severe disability & $73(26.1)$ & $56(24.1)$ & $17(35.4)$ & \\
\hline Moderate disability & $66(23.6)$ & $58(25.0)$ & $8(16.7)$ & \\
\hline Good recovery & $78(27.9)$ & $64(27.6)$ & $14(29.2)$ & \\
\hline \multicolumn{5}{|l|}{ Mean monitored signals \pm SD } \\
\hline $\mathrm{ABP}(\mathrm{mm} \mathrm{Hg})$ & $91.31 \pm 12.15$ & $91.09 \pm 12.57$ & $92.36 \pm 9.91$ & 0.510 \\
\hline $\mathrm{ICP}(\mathrm{mm} \mathrm{Hg})$ & $17.85 \pm 9.07$ & $16.99 \pm 9.29$ & $21.98 \pm 6.58$ & $<0.001$ \\
\hline $\mathrm{CPP}(\mathrm{mm} \mathrm{Hg})$ & $73.46 \pm 12.25$ & $74.10 \pm 12.42$ & $70.39 \pm 11.01$ & 0.056 \\
\hline $\mathrm{FV}(\mathrm{cm} / \mathrm{sec})$ & $64.08 \pm 24.89$ & $64.74 \pm 25.94$ & $60.93 \pm 18.96$ & 0.336 \\
\hline
\end{tabular}

Following the introduction of nICP, eCPP (in $\mathrm{mm} \mathrm{Hg}$ ) can then be rendered as:

$$
e C P P=A B P-n I C P
$$

or

$$
e C P P=A B P \cdot\left[0.734-\frac{0.266}{\sqrt{(C V R \cdot C a \cdot H R \cdot 2 \pi)^{2}+1}}\right]-7.026 \quad[\text { Eq. 2] }
$$

In this way, eCPP is associated with physiological parameters of CVR, compliance, and heart rate, with ABP and $\mathrm{FV}$ as the required measurements. The performance of the new estimator was tested by comparing eCPP against invasive $\mathrm{CPP}$, using data from the validation group.

The dichotomization of the total population of patients into 2 groups (formation and validation) was based on an optimization procedure for exploiting in full the available set of data. For this reason, a simple minimum requirement criterion was set, in which the validation group included only patients with a minimum of 5 or more recording sessions per patient. This selection subsequently dictated the number of patients to be included in the second group, used for formation of eCPP. Dichotomizing the cohort of patients in this way presented 2 benefits: 1) the multirecording validation portion of the study allowed for the determination of the correlation between eCPP and $\mathrm{CPP}$ using repetitive measurements from individual patients, and 2) the high number of recordings included in the formation group increased the statistical power of the

TABLE 2. Summary of regression analysis parameters*

\begin{tabular}{lccc}
\hline Variable & Coefficients & SEM & $95 \% \mathrm{Cl}$ \\
\hline CrCP & 0.266 & 0.035 & $0.198-0.333$ \\
\hline Constant & 7.026 & 1.361 & $4.352-9.700$ \\
\hline
\end{tabular}

* Model summary (nICP): $R=0.340, F=59.225, p<0.001$. regression model, hence rendering a more accurate eCPP. A full overview of the terminology used in this study is presented in Table 3.

\section{Statistical Analysis}

Statistical analysis of the data was conducted with SPSS statistical software (version 20, IBM). The analysis included bivariate correlations, with $\mathrm{R}$ representing the Pearson correlation coefficient, and the level of significance ( $\mathrm{p}$ value) set at 0.05 . Results are presented in a mean

TABLE 3. Terminology of used parameters

\begin{tabular}{lll}
\hline Symbol & \multicolumn{1}{c}{ Term } & \multicolumn{1}{c}{ Units } \\
\hline ABP & Arterial blood pressure & $\mathrm{mm} \mathrm{Hg}$ \\
\hline $\mathrm{Ca}$ & Cerebral arterial compliance & $\mathrm{cm} / \mathrm{mm} \mathrm{Hg}$ \\
\hline $\mathrm{CaBV}$ & Cerebral arterial blood volume & $\mathrm{cm}^{3}$ \\
\hline $\mathrm{CBF}$ & Cerebral blood flow & $\mathrm{cm}^{3} / \mathrm{sec}$ \\
\hline $\mathrm{CPP}$ & Cerebral perfusion pressure & $\mathrm{mm} \mathrm{Hg}$ \\
\hline $\mathrm{CrCP}$ & Critical closing pressure & $\mathrm{mm} \mathrm{Hg}$ \\
\hline $\mathrm{CrCPi}$ & Invasive model of critical closing pressure & $\mathrm{mm} \mathrm{Hg}$ \\
\hline $\mathrm{CVR}$ & Noninvasive cerebrovascular resistance & $\mathrm{mm} \mathrm{Hg} /(\mathrm{cm} / \mathrm{sec})$ \\
\hline $\mathrm{CVRi}$ & Invasive cerebrovascular resistance & $\mathrm{mm} \mathrm{Hg} /(\mathrm{cm} / \mathrm{sec})$ \\
\hline $\mathrm{eCPP}$ & Noninvasive estimator of CPP & $\mathrm{mm} \mathrm{Hg}$ \\
\hline $\mathrm{FV}$ & Blood flow velocity & $\mathrm{cm} / \mathrm{sec}$ \\
\hline $\mathrm{HR}$ & Heart rate & $\mathrm{beat} / \mathrm{min}$ \\
\hline $\mathrm{ICP}$ & Intracranial pressure & $\mathrm{mm} \mathrm{Hg}$ \\
\hline $\mathrm{nICP}$ & Noninvasive estimator of ICP & $\mathrm{mm} \mathrm{Hg}$ \\
\hline $\mathrm{Sa}$ & Cross-sectional area of the insonated & $\mathrm{cm}{ }^{2}$ \\
& vessel & \\
\hline TAU & Time constant of the cerebrovascular & $\mathrm{sec}$ \\
& arterial bed & \\
\hline
\end{tabular}


\pm SD format. Normal distribution was established with the Shapiro-Wilk test. The Bland-Altman method was used to determine the agreement between measured parameters and their noninvasive estimations. A receiver operating characteristic (ROC) was further used to determine the ability of eCPP to predict CPP, presented with areas under the curve (AUC); the predicting ability is considered reasonable when the AUC is higher than 0.7 and strong when the AUC exceeds 0.8. ${ }^{16}$ The classification decision tree was created with the Chi-squared Automatic Interaction Detection (CHAID) growing method.

\section{Results}

\section{Comparison of eCPP to Measured CPP for Patients as a Cohort}

When we assessed the group of patients as a cohort, the averaged per patient eCPP was strongly correlated with the invasively measured CPP $(\mathrm{R}=0.851, \mathrm{p}<0.001, \mathrm{n}=48$; Fig. 1 left), with a mean \pm SD difference between CPP and eCPP of $4.02 \pm 6.01 \mathrm{~mm} \mathrm{Hg}$ (Fig. 1 right). In total, $83.3 \%$ of the cases (40 patients) had an absolute estimation error of eCPP to CPP below $10 \mathrm{~mm} \mathrm{Hg}$.

The eCPP maintained its estimation accuracy when we assessed the group of 48 patients as a cohort of recordings $(\mathrm{n}=325)$, with a correlation to CPP of $\mathrm{R}=0.813$, a mean difference of $3.70 \pm 8.70 \mathrm{~mm} \mathrm{Hg}$, and with $259(79.7 \%)$ of 325 recordings with an absolute estimation error less than $10 \mathrm{~mm} \mathrm{Hg}$ (IQR 2.47-6.09 mm Hg).

Further analysis of the recordings with a classification decision tree indicated the absolute estimation errors for various ranges of CPP: for CPP greater than $80.19 \mathrm{~mm} \mathrm{Hg}$, the absolute estimation error of eCPP was $4.31 \pm 2.86 \mathrm{~mm}$ $\mathrm{Hg}$ (97 recordings), followed by a range of CPP between 63.01 and $80.19 \mathrm{~mm} \mathrm{Hg}$, which had an absolute estimation error of $5.55 \pm 4.26 \mathrm{~mm} \mathrm{Hg}$ (131 recordings). The distribution of the absolute error of eCPP - CPP across the range of CPP values is demonstrated in Fig. 2.

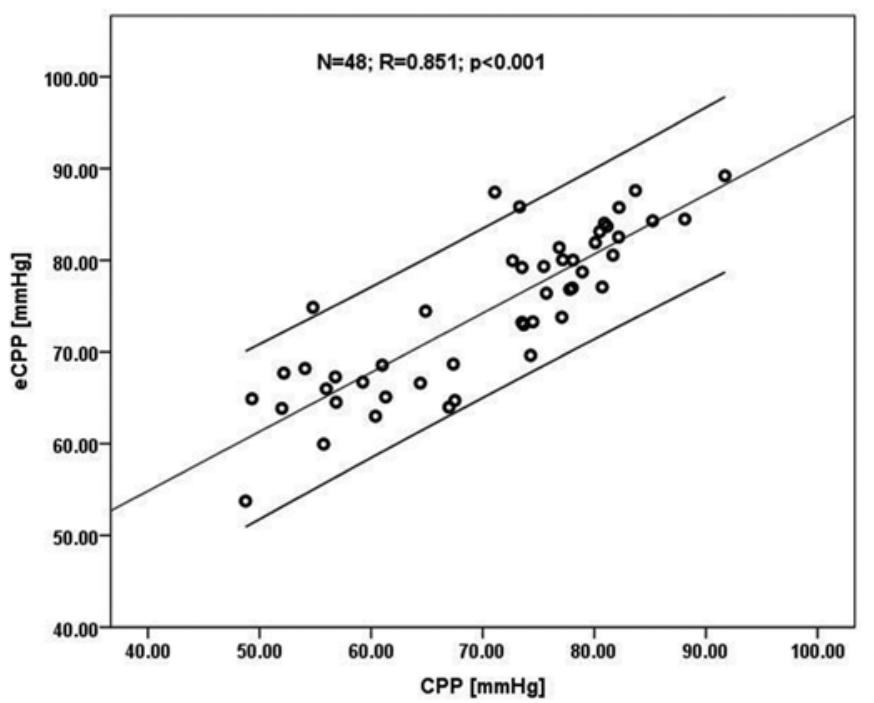

\section{Low CPP Prediction Analysis}

An ROC curve analysis was used to determine the ability of eCPP to predict a low value of CPP in 325 recordings. Three different limits were set for CPP: 50, 60, and $70 \mathrm{~mm} \mathrm{Hg}$ (Table 4). In this analysis, eCPP accurately predicted low CPP, with an AUC greater than 0.8 for all 3 limits (Table 4, Fig. 3).

\section{Temporal Analysis of eCPP as an Estimator of Invasive CPP}

In temporal analysis, each patient was assessed individually, rendering an individual correlation coefficient and a mean \pm SD difference of eCPP from CPP between recording sessions. These individual results were then averaged for all patients in the application group, resulting in an eCPP that was found to be strongly correlated with $\mathrm{CPP}$ (mean $\mathrm{R}=0.733$, range $0.231-0.993$ ). Examples of individual correlations between eCPP and CPP for patients are presented in Fig. 4.

For each patient, eCPP presented a mean difference from CPP of $3.45 \mathrm{~mm} \mathrm{Hg}$ (range -4.69 to $19.03 \mathrm{~mm} \mathrm{Hg}$ ), and a mean SD of this difference of $5.52 \mathrm{~mm} \mathrm{Hg}$ (range $1.52-10.76 \mathrm{~mm} \mathrm{Hg}$ ). These data imply a $95 \% \mathrm{CI}$ of the SD for estimating CPP within 1 recording session of a patient to be $1.89-5.01 \mathrm{~mm} \mathrm{Hg}$.

Examples of how eCPP approximates changes of CPP in various scenarios captured during recording sessions of patients with TBI are presented in Fig. 5. Figure 6 further demonstrates in detail how the calculation of eCPP is affected by changes in its input parameters, consisting of monitored signals and estimated modules, during an event of arterial hypotension in a recording session of a single patient.

\section{Discussion}

We have constructed a new model for a noninvasive assessment of CPP based on CrCP, requiring only ABP and TCD measurements, the combination of which is attempt-

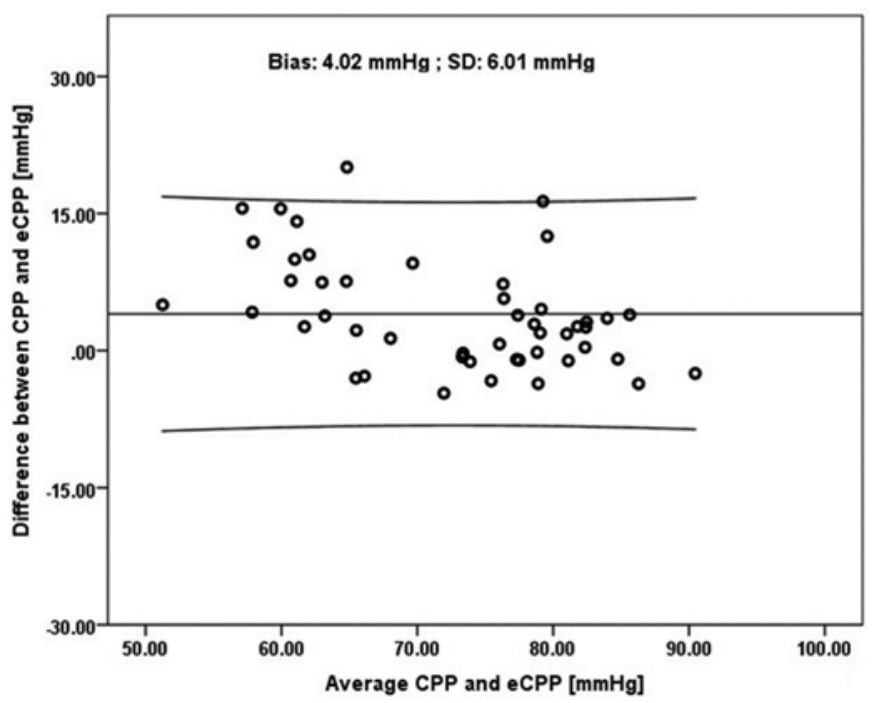

FIG. 1. Left: Scatterplot showing the correlation of the noninvasive methodology for assessing CPP, noted as eCPP, with measured CPP. Right: Bland-Altman plot for comparing differences between CPP and eCPP for average values of CPP and eCPP. Each point represents 1 patient. Outer thick lines represent the $95 \% \mathrm{Cl}$. 


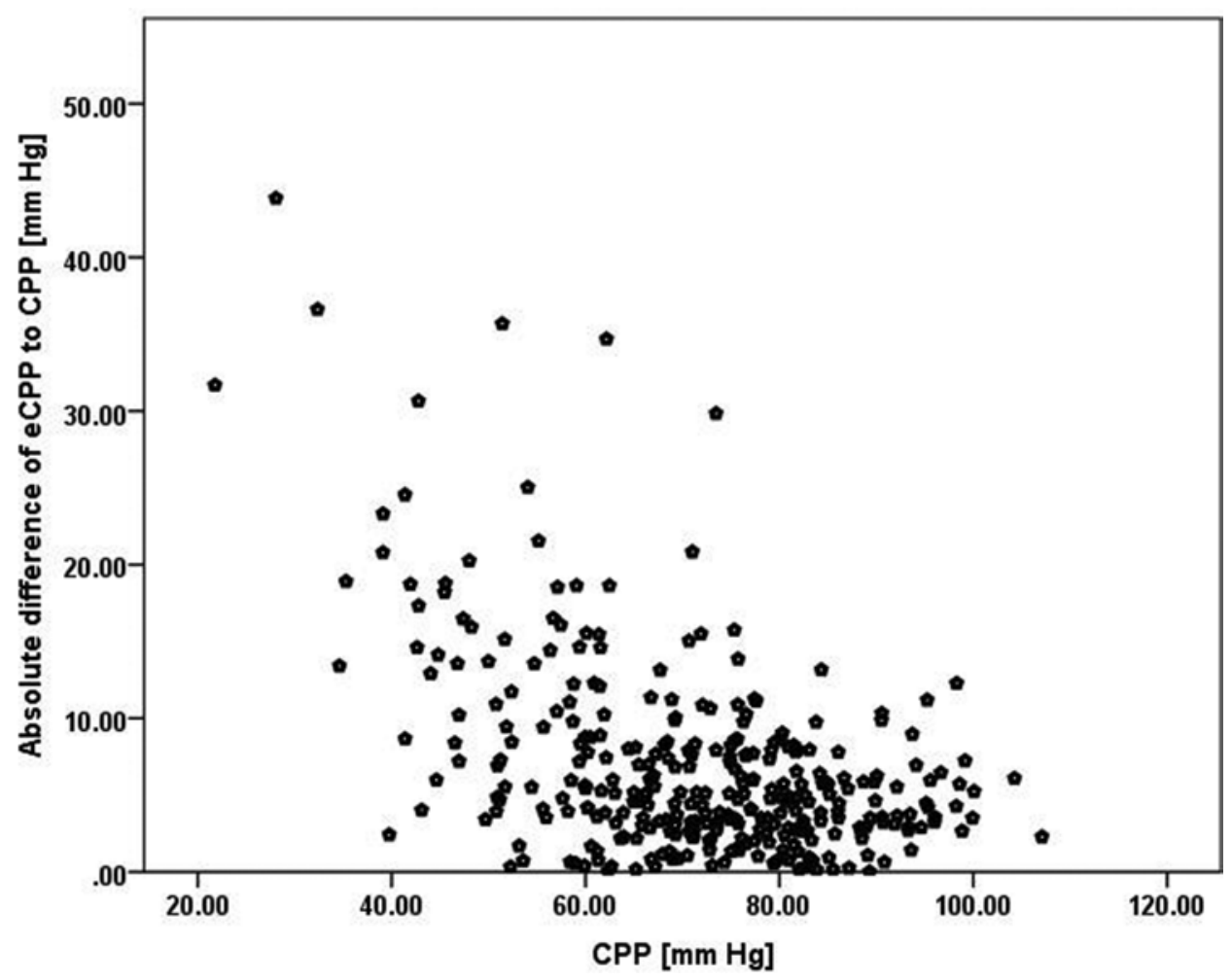

FIG. 2. Scatterplot showing the distribution of absolute error of CPP - eCPP across the range of CPP values, with each point representing 1 recording session (325 recordings in total). Estimation of CPP is shown to be most accurate in the range of 60 to 90 $\mathrm{mm} \mathrm{Hg}$, presented with decreasing absolute difference to CPP.

ing to compensate for exclusion of invasive ICP measurements. Assessing CPP noninvasively can be beneficial in many clinical scenarios in which ICP monitoring is not indicated or is unavailable: 1) in patients with borderline indications for invasive ICP measurements based on Brain Trauma Foundation guidelines; ${ }^{7}$ 2) in patients with coagulopathy in trauma/hepatic encephalopathy; and 3) in polytrauma patients in an emergency, where a rapid assessment of CPP status is required during treatment of other injuries.

The basis of the new CPP estimator lies in a complex mathematical model, which takes into account physiological parameters derived from ABP and FV signals, such as cerebrovascular compliance, resistance, and heart rate. The formation of eCPP methodology required an interim step of assessing ICP with a noninvasive predictor model, built with the use of a regression analysis with $\mathrm{CrCP}$.

The validation of eCPP presented in this study included comparison analysis relative to invasively measured CPP, both at a level of a cohort analysis and at an individual level in which each patient's monitoring session was examined individually across time.

TABLE 4. Comparison of predictive power of eCPP for different levels of CPP, as assessed with ROC curve analysis and presented with AUC

\begin{tabular}{cc}
\hline CPP Limit $(\mathrm{mm} \mathrm{Hg})$ & eCPP AUC $(95 \% \mathrm{Cl})$ \\
\hline$<50$ & $0.905(0.860-0.950)$ \\
\hline$<60$ & $0.889(0.849-0.929)$ \\
\hline$<70$ & $0.913(0.883-0.944)$ \\
\hline
\end{tabular}

\section{Cerebral Perfusion Pressure as a Number}

Many clinical protocols for post-head-injury management are focused on guidelines requiring a range or an optimum value of CPP that needs to be achieved for an adequate perfusion of the brain., ${ }^{7,912-14,22,23,31,33,35}$ For this reason, we sought to determine the accuracy of estimating $\mathrm{CPP}$ as a number by examining the group of patients as an averaged cohort.

The results indicated that measurements obtained with eCPP were highly correlated with those for invasive CPP, with a mean \pm SD difference from CPP of $4.02 \pm 6.01 \mathrm{~mm}$ $\mathrm{Hg}$. In terms of absolute error, CPP was estimated by eCPP with an error margin below $10 \mathrm{~mm} \mathrm{Hg}$ in more than $80 \%$ of the analyzed cases, with eCPP demonstrated to be most accurate in the CPP range of 60 to $90 \mathrm{~mm} \mathrm{Hg}$ (Fig. 2). Even though there is a lack of consensus regarding the ideal range of CPP after TBI, and the error difference of more than $10 \mathrm{~mm} \mathrm{Hg}$ may or may not be clinically relevant depending on the duration of such a discrepancy, ${ }^{4,41}$ both the normal CPP range of $70-85 \mathrm{~mm} \mathrm{Hg}^{41}$ and suggestions for maintaining a CPP of at least $70 \mathrm{~mm} \mathrm{Hg}$ after head injury, ${ }^{35}$ or between 50 and $70 \mathrm{~mm} \mathrm{Hg}, 7$ or at a static autoregulation range (i.e., $60-70 \mathrm{~mm} \mathrm{Hg}$ ) ${ }^{12}$ are within the range of the highest accuracy of eCPP. This ability of eCPP to estimate CPP most accurately at an expected or suggested level of CPP seems promising for clinical use of the method.

The outcome of a patient following TBI has been known to be significantly affected by exposures to secondary insults such as systemic hypotension or intracranial hypertension, which can result in severely reduced 

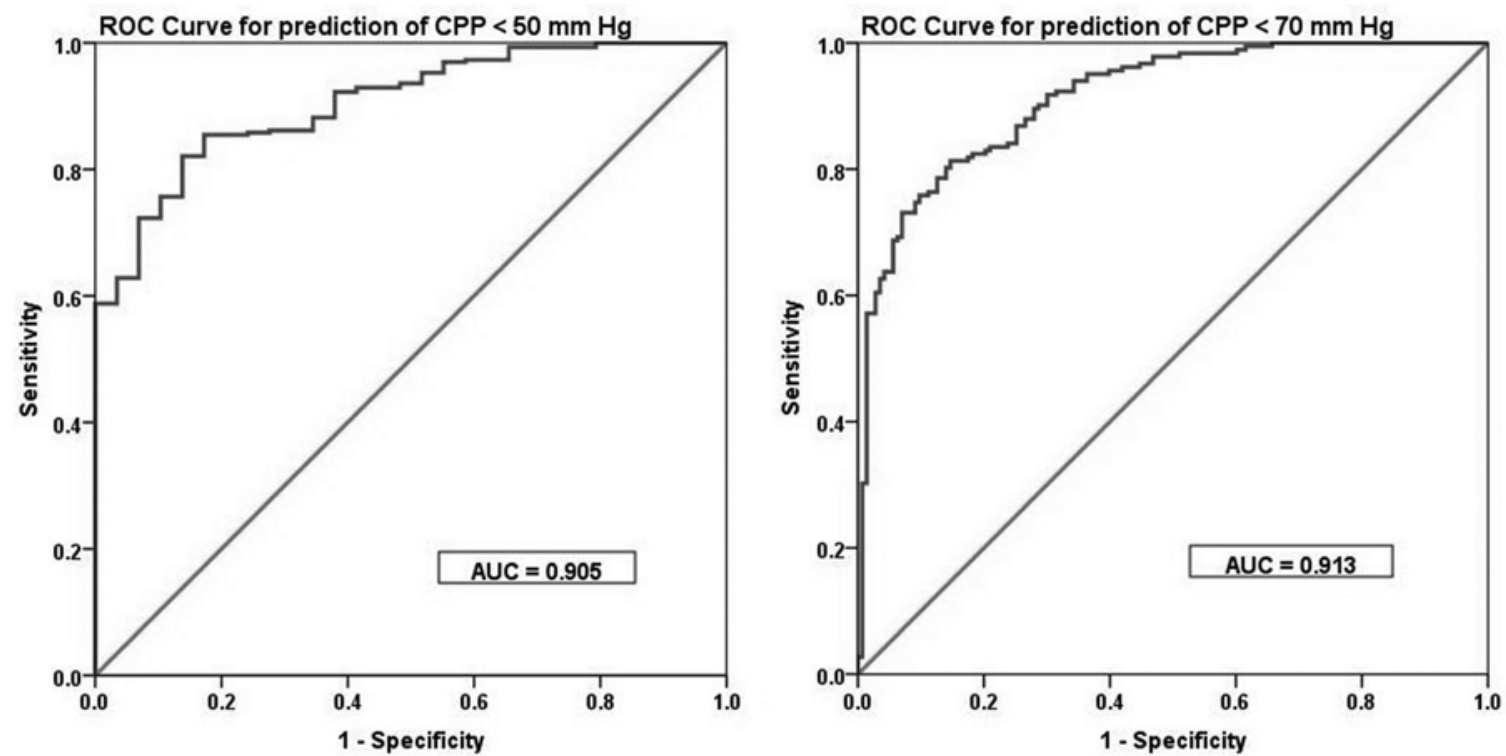

FIG. 3. ROC curve analyses for predicting low CPP with eCPP for 2 different thresholds of CPP: $50 \mathrm{~mm} \mathrm{Hg}$ (left) and $70 \mathrm{~mm} \mathrm{Hg}$ (right). In both cases, eCPP demonstrated a strong predictive power, indicated by the AUC values.

CPP and cerebral ischemia ${ }^{6,29}$ when CPP decreases below 50-60 mm Hg. ${ }^{41}$ The eCPP demonstrated a strong ability to predict low values of CPP, as examined in 3 different thresholds for CPP values below 50,60, and $70 \mathrm{~mm} \mathrm{Hg}$. Therefore, this signifies that eCPP could act as a noninvasive indicator of potentially inadequate $\mathrm{CPP}$, acting as a warning for hypoperfusion during monitoring of a patient.

\section{Cerebral Perfusion Pressure as a Time-Varying Variable}

Apart from assessing CPP solely as a number, some studies suggest that CPP is better considered as a condition of $\mathrm{CBF}$, reflecting the hemodynamic status of a patient after TBI. ${ }^{10,21,37}$ This suggestion is based on the fact that in clinical practice, brain perfusion assessed through CPP can be underestimated due to ABP monitoring devices
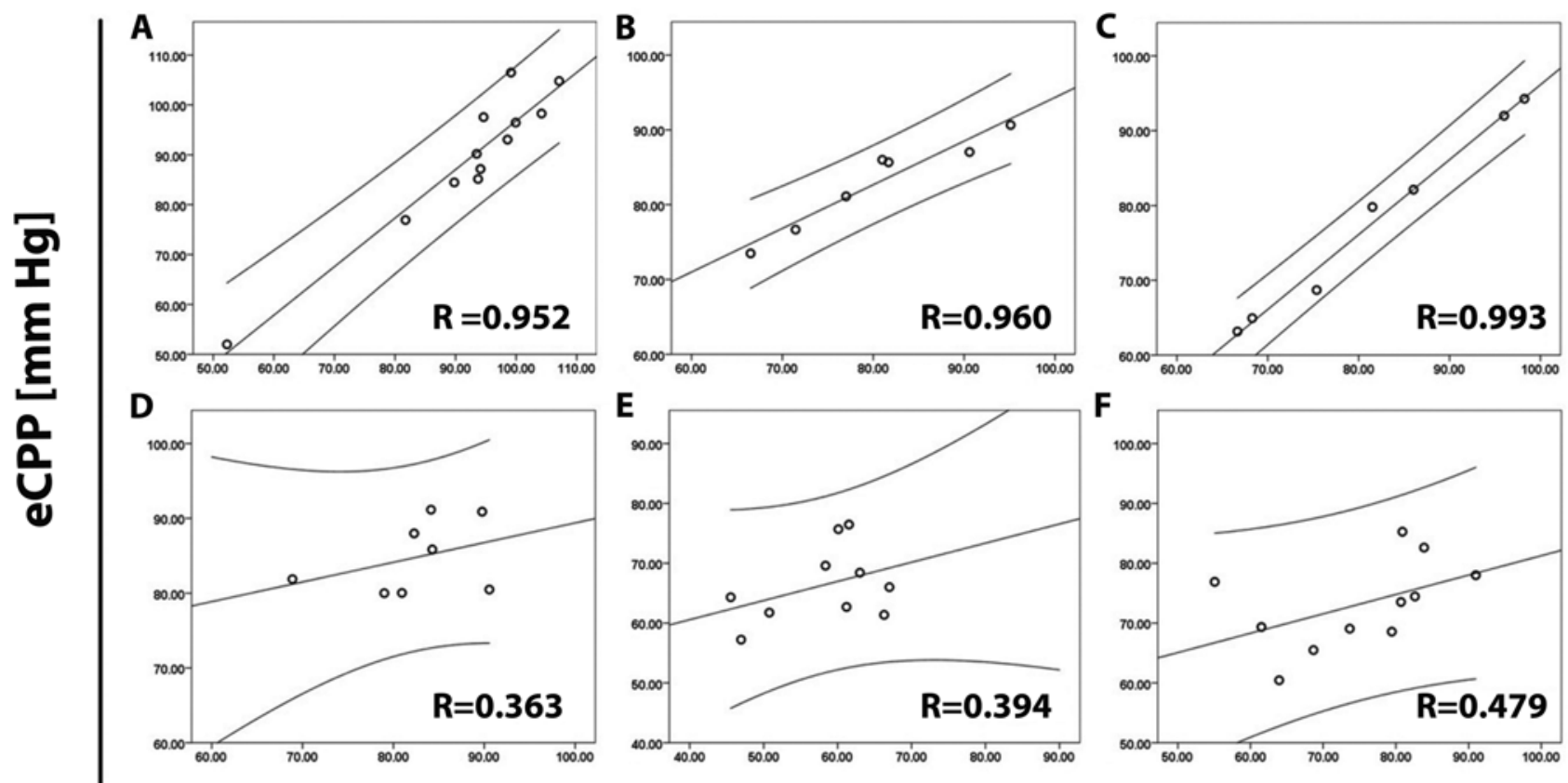

\section{CPP [mm Hg]}

FIG. 4. Examples of individual correlations between CPP and eCPP. Each graph represents 1 patient and each open circle represents 1 recording session. The upper row $(\mathrm{A}-\mathrm{C})$ demonstrates cases of good correlations between $\mathrm{CPP}$ and eCPP, whereas the lower row (D-F) presents the weaker cases. Weaker cases are associated with overestimation of CPP. 


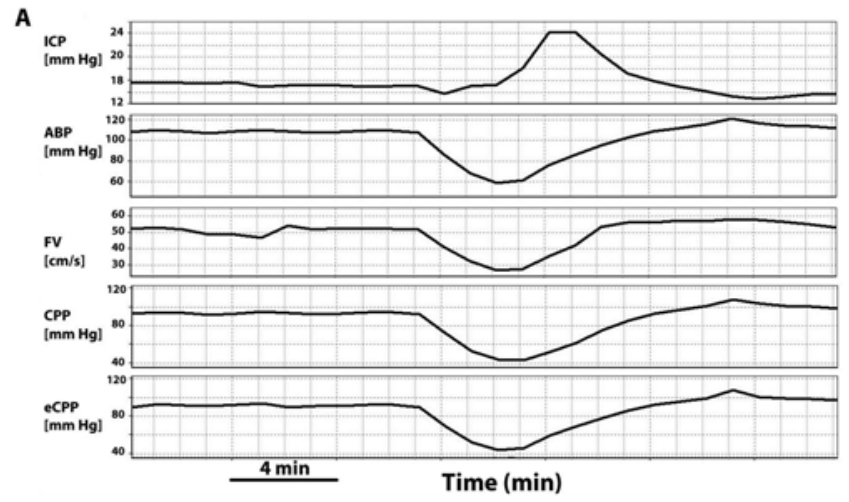

B

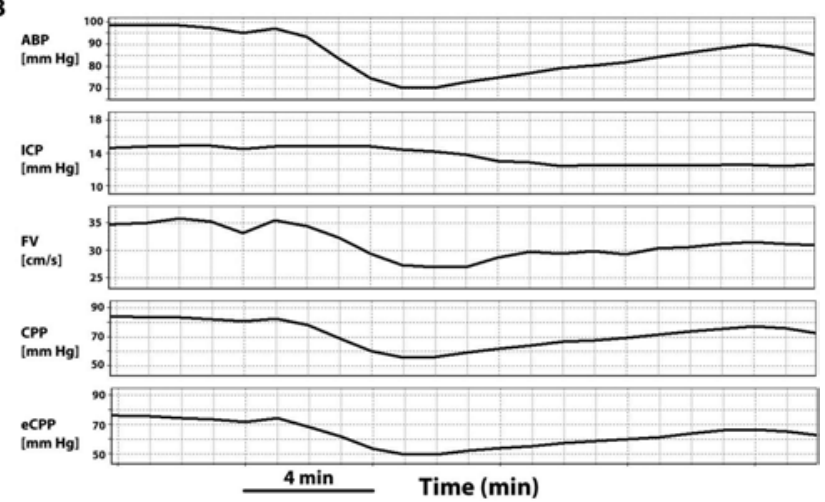

C

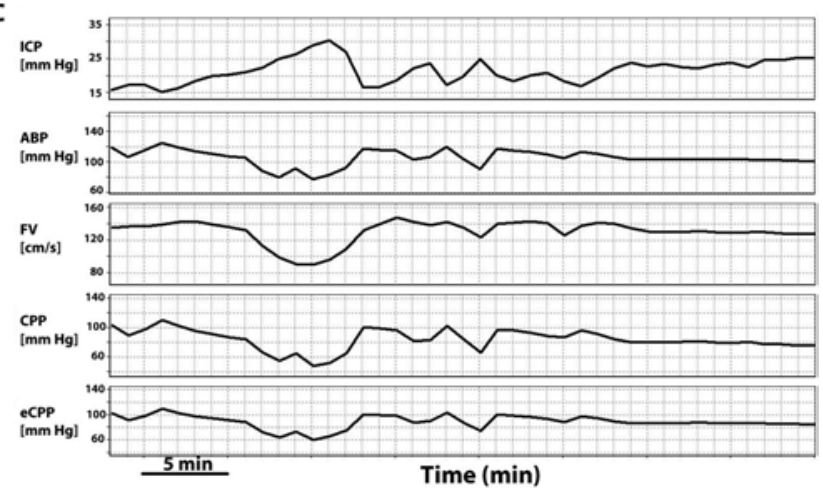

FIG. 5. Examples of how CPP was approximated by eCPP in various CPP-affecting phenomena that occurred during monitoring. In all cases, changes in CPP are depicted by eCPP. A: Incidental decrease in ABP causing a temporal rise in ICP and a reduction in CPP and blood FV. B: Arterial hypotension resulting in a decrement of CPP and FV with unchanged ICP. C: Fluctuations of CPP.

measuring readings from peripheral vessels and calibrated at the heart level and not at the head level. ${ }^{10}$ Therefore, brain perfusion is better represented by global CBF assessed through MRI or PET, instead of a numerical value of CPP. However, these existing methods for accurately assessing CBF are not suitable for continuous monitoring, ${ }^{37}$ the role of which is thereby served by CPP. A CPP that is considered not only as a value but rather as a time variable can then indicate changes in $\mathrm{CBF}$ over time, therefore being able to characterize changing hemodynamic needs, such as in cases of arterial hypotension or intracranial hypertension insults that would threaten to reduce CBF.

In this part of our study, each patient was assessed in- dividually, with the accuracy levels of eCPP improving in comparison with the respective cohort analysis (mean \pm SD differences from CPP of $3.45 \pm 5.52 \mathrm{~mm} \mathrm{Hg}$ vs $4.02 \pm$ $6.01 \mathrm{~mm} \mathrm{Hg}$, respectively). This finding implies that eCPP provides a more accurate $\mathrm{CPP}$ estimation when $\mathrm{CPP}$ is assessed over time, i.e., for following changes in CPP during a monitoring session when ABP and TCD measurements are performed. The underlying factors for this difference might have to do with how ABP- and TCD-derived information is being exploited; in cohort analysis the information regarding $\mathrm{ABP}$ and $\mathrm{FV}$ dynamics, as contained in $\mathrm{CrCP}$, is averaged out, thereby potentially "hiding" phenomena that could influence CPP for a short period of time. In contrast, in time analysis these phenomena, such as a drop in ABP, are captured, therefore improving the accuracy of eCPP. This was further demonstrated in the examples shown in Fig. 5, in which eCPP correctly depicted a variety of changes and fluctuations of CPP.

\section{The Role of ICP}

Intracranial pressure has an important role in TBI management, as phenomena of intracranial hypertension can lead to a decrement in CPP and a potential decrease in CBF, causing secondary ischemic insults. ${ }^{15,23,26,39}$ In fact, many of the studies recommending CPP thresholds are confounded by high ICP, therefore further signifying its clinical role. However, focusing solely on ICP would not be sufficient, as from a physiological viewpoint, both individual components of CPP (ICP and ABP) have been shown to affect $\mathrm{CBF}$ and consist of independent predictors of outcome after TBI. ${ }^{6,15,29,31,39}$ Hence, monitoring and management of CPP will remain one of the cornerstones of TBI management, even though it is recognized that further work is required in terms of patient-specific thresholds. ${ }^{4,41}$ For this reason, the development of noninvasive methods to monitor CPP is reasonable and could provide an additional option in resource-limited settings or for patients who have contraindications for invasive monitoring.

The invasive character of ICP has also been the limiting factor for these noninvasive CPP assessments, with high ICP resulting in overestimation of CPP. Therefore, information derived from both ABP and TCD measurements is being used, attempting to compensate for the absence of invasive ICP measurements. ${ }^{2,5,10,18,38}$ In contrast to other methodologies, the basis of the eCPP method consists of a regression analysis, constructed with a large number of TBI recordings (455 in total), which included cases in which ICP is either low $(5 \mathrm{~mm} \mathrm{Hg}$ ) or very high (above $40 \mathrm{~mm} \mathrm{Hg}$ ). In this matter, eCPP contains some TCD- and ABP-derived descriptive information about these cases, encapsulated in the form of cerebrovascular compliance and resistance in CrCP. This aided eCPP in capturing circumstances of low and very low CPP, demonstrated with high levels of sensitivity and specificity for detecting reduced CPP. Even though high ICP does cause a CPP overestimation by eCPP, restricting its capability in terms of estimating CPP as an absolute number, the loss of precision at high ICP is mitigated by its ability to detect low CPP.

\section{Benefits of Using CrCP for CPP Estimation}

A benefit of using impedance $\mathrm{CrCP}$ for estimating CPP 


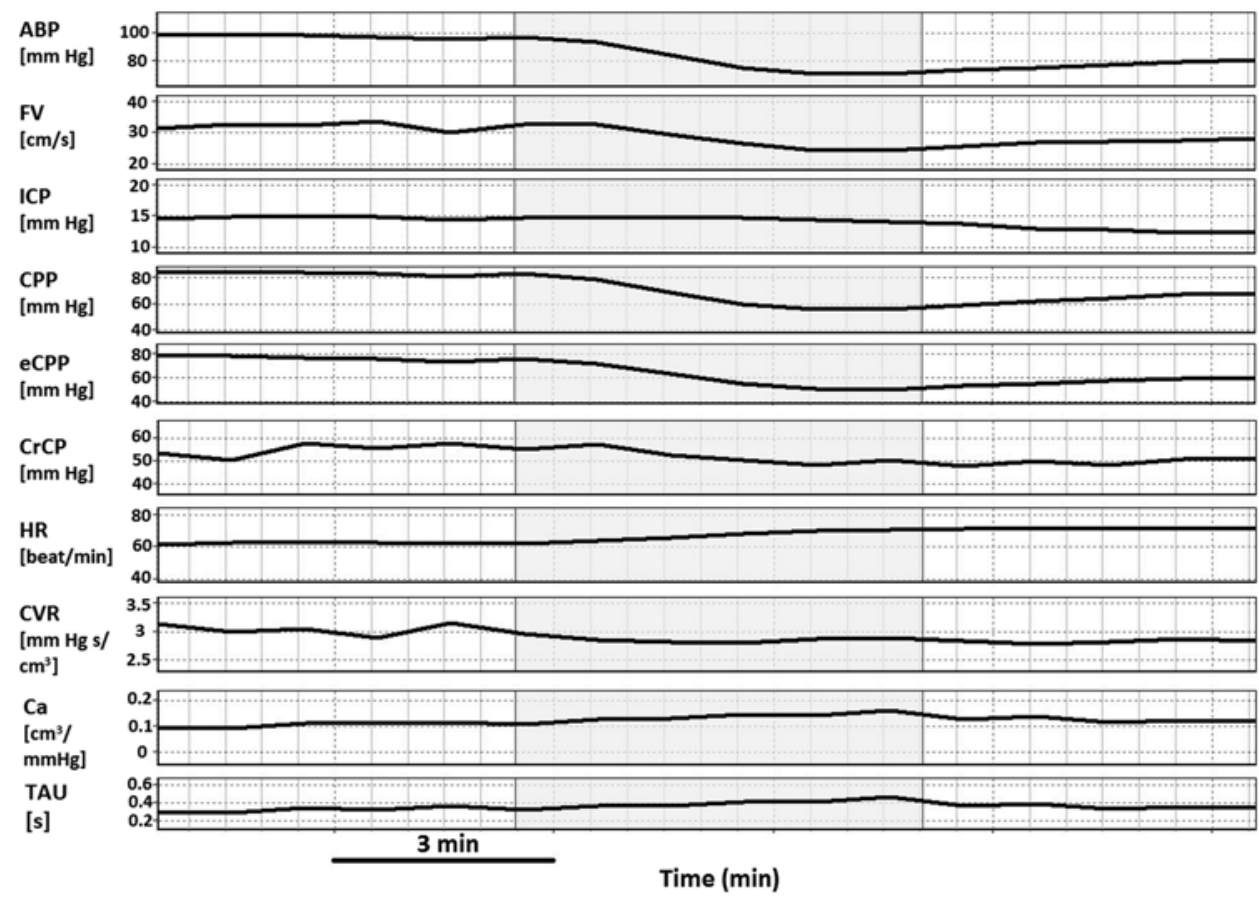

FIG. 6. Example of how monitored signals and estimated parameters affect the calculation of eCPP during an event of arterial hypotension (gray area) in a recording session of a single patient. Monitored signals included ABP, TCD blood FV, and ICP, while estimated parameters included CPP, CVR, compliance (Ca), and time constant (TAU). A decrease in ABP with an increase in heart rate $(H R)$ is followed by compensatory vasodilatation, reducing CVR and increasing compliance, which in turn leads to an increase in TAU and a reduction in $\mathrm{CrCP}$ due to decreased vasomotor tone of the small cerebral vessels. Overall, changes in the variables of the eCPP formula (see Appendix) ABP, HR, CVR, and compliance (or TAU being the product of the latter 2 variables) led to eCPP presenting an estimated decrease in CPP.

is that it allowed us to associate the CPP estimator with further physiological parameters such as heart rate and measures of the resistance and compliance of the cerebrovascular bed. eCPP subsequently contains the product of CVR and compliance, relating eCPP to the physiological measurement of TAU, which is an estimate of how fast the cerebral arterial bed is filled by blood volume after a sudden change in ABP during 1 cardiac cycle. ${ }^{19}$ Due to this relationship, eCPP as a function of TAU and heart rate can then take into account changes in primary variables describing cerebral hemodynamics and cardiac function, enhancing its physiological presence for estimating CPP.

A further advantage of having TAU in the eCPP formula is the inherited independence of eCPP from the unknown cross-sectional area of the insonated vessel, as it gets eliminated through TAU calculation (see Appendix). ${ }^{19}$

\section{Limitations of the Study}

The calculations regarding $\mathrm{CrCP}$ and consequently eCPP depended on information derived from ABP and FV. Measurement and monitoring of these 2 parameters pose some limitations in regard to the accuracy of the estimations. First, a good quality of recording is required, and in regard to the TCD technique, the quality depends, among other parameters, on the experience of the user for accurately insonating the targeted artery (the MCA). Moreover, unlike ABP measurements, TCD monitoring was not continuous but instead it included short recording periods for every patient on a daily basis, therefore posing a restriction on continuous eCPP assessment. The reasons behind short recordings were: 1) the needs of a neurointensive care environment, in which treatment of headinjured patients includes change in position or transfer (for CT/MRI scan purposes) resulting in an unavoidable interruption of TCD recordings; and 2) the limitations of the current TCD technology, regarding the limited capability of probe holders, which are not well suited for prolonged continuous recording. An expected improvement in TCD technology for continuous FV monitoring would allow full exploitation of the eCPP methodology in the future (http://www.prnewswire.com/news-releases/physio sonics-announces-fda-clearance-for-presto-1000-flowmonitor-for-ultrasound-cranial-monitoring-184153001. html).

The use of radial artery ABP zeroed at the level of the heart instead of actual blood pressure in the brain could be considered a limitation of the study, in terms of how well the level of peripheral ABP can approximate the respective intracranial ABP. Heart-level calibration leads to an overestimation of actual CPP at the head level, ${ }^{17}$ with this difference also affecting the impedance calculation that derives information from $\mathrm{ABP}$ measurements. However, this limitation may not have been too significant in this study because the primary comparisons between the invasive (CPP) and noninvasive calculations had a common basis, the same ABP point of measurement. Therefore, this limi- 
tation may not have affected the actual aims of this study; as long as the same level of ABP measurement is used, a similar estimation accuracy of CPP can be expected.

The comparison of eCPP with CPP could also be applied to a comparison between invasive ICP and nICP, as mathematically the two models (nICP and eCPP) differ only in terms of the addition of ABP in eCPP. However, even though the accuracy levels for CPP estimation are promising, a similar accuracy for ICP may not be adequate, due to the difference in magnitude between CPP and ICP. An estimation error of 5-10 $\mathrm{mm} \mathrm{Hg}$ could be clinically acceptable for CPP, ranging, for example, from 60 to $90 \mathrm{~mm} \mathrm{Hg}$, but would not be sufficient for an ICP ranging from 5 to $25 \mathrm{~mm} \mathrm{Hg}$. Even though this would limit the use of $\mathrm{CrCP}$ for noninvasive ICP estimation, it allowed the formation of the eCPP model, which rendered encouraging results for its use in clinical practice.

The number of patients in whom the eCPP methodology was applied might pose a limitation in this study, regarding the coverage of different clinical scenarios with a small sample consisting of 48 patients. However, these patients had a high number of repetitive measurements $(\geq$ 5 per patient), therefore fulfilling the purpose of the cohort's dichotomization regarding the validation of eCPP as an accurate estimator of CPP, examined as a time-varying variable. An overall synopsis of the advantages and limitations of using eCPP is presented in Table 5.

\section{Conclusions}

The use of CrCP formed the basis of a new methodology that demonstrated promising results in regards to a noninvasive estimation of CPP. Analysis of patients as a cohort and through individual recording sessions resulted in a strong correlation between eCPP and CPP, presenting a relatively small estimation error. Most importantly, low values of CPP were well detected by eCPP, which showed strong predictive power and the ability to act as a noninvasive indicator of low or inadequate perfusion.

\section{Appendix}

\section{Calculation of Noninvasive Critical Closing Pressure (CrCP)}

The basis for the noninvasive model is given by the invasive multiparameter impedance method: ${ }^{39}$

$$
C r C P i=A B P-\frac{C P P}{\sqrt{(C V R i \cdot C a \cdot H R \cdot 2 \pi)^{2}+1}} \quad[\mathrm{~mm} \mathrm{Hg}]
$$

In this model, the invasive parts of the formula (containing the ICP parameter) are CPP and invasive cerebrovascular resistance (CVRi), which can be approximated as: ${ }^{20}$

$$
C V R i=\frac{C P P}{F V \cdot S a} \quad\left[\frac{\mathrm{mm} \mathrm{Hg} \cdot \mathrm{s}}{\mathrm{cm}^{3}}\right]
$$

In this equation, the parameter $\mathrm{Sa}$ in the denominator represents the unknown cross-sectional area of the insonated vessel.

Ca denoting the compliance of the cerebrovascular bed can be estimated as: $:^{20}$

$$
C a=\frac{C a B V 1 \cdot S a}{A 1} \quad\left[\frac{\mathrm{cm}^{3}}{\mathrm{~mm} \mathrm{Hg}}\right]
$$

In this equation, $\mathrm{A} 1$ is the fundamental harmonic amplitude of $\mathrm{ABP}$, whereas $\mathrm{CaBV} 1$ is the amplitude of the fundamental harmonic of cerebral arterial blood volume (CaBV), derived by using a 10 -second discrete Fourier transformation of CaBV's time series. Changes of pulsatile $\mathrm{CaBV}$ in turn can be approximated by integrating the FV pulse waveform with the beat-to-beat mean removed, in the form of samples of instant and average values of FV, respectively: ${ }^{20}$

$$
\Delta C a B V(n)=S a \cdot \sum_{i=1}^{n}(F V(i)-m e a n F V) \Delta t
$$

In this equation, $\mathrm{n}$ is the number of the samples and $\Delta \mathrm{t}$ is the time interval between 2 consecutive samples. Instant (sampled) arterial blood flow velocity is represented by FV(i), while mean FV represents the corresponding average value.

By having the product of $\mathrm{Ca}$ and CVRi in Eq. A, the parameter $\mathrm{Sa}$ is cancelled out, as has been described in the impedance methodology. ${ }^{39}$

TABLE 5. Advantages and disadvantages of using eCPP

Advantages

The estimation error of eCPP is relatively small, hence being able to provide an indication of CPP levels where invasive measurements are not available

eCPP is strongly correlated with invasive CPP, presenting a good indication of changes in CPP over time

Low values of CPP are well detected by eCPP, highlighting its ability to act as a noninvasive indicator of low or inadequate perfusion

Even though there is a lack of consensus regarding an ideal range of CPP after TBI, all the guideline levels are within the range of highest accuracy of eCPP

eCPP has a physiological substance, as its estimation is based on physiological parameters of heart rate, cerebrovascular resistance, compliance, and cerebral time constant

eCPP is not affected by a common limitation of measuring blood flow through TCD ultrasonography as its calculation is independent of the unknown cross-sectional area of the insonated vessel, i.e., the MCA during measurement periods

Disadvantages

Requires TCD measurements that are hard to obtain continuously for long-term monitoring of eCPP

Requires suitable software for a next-to-bed instant calculation, based on the monitored signals of ABP and FV

High ICP causes a CPP overestimation by eCPP, restricting its capability in terms of estimating CPP as an absolute number. However, the loss of precision at high ICP is mitigated by eCPP's ability to detect low and very low CPP 
In order to create the noninvasive version of $\mathrm{CrCPi}$, we approximate CPP with ABP and thus CVRi can be now approximated noninvasively as:11

$$
C V R=\frac{A B P}{F V \cdot S a} \quad\left[\frac{\mathrm{mm} \mathrm{Hg} \cdot \mathrm{s}}{\mathrm{cm}^{3}}\right]
$$

Based on these remarks, the noninvasive model of impedance $\mathrm{CrCP}$ (in $\mathrm{mm} \mathrm{Hg}$ ) is given as:

$$
C r C P=A B P \cdot\left[1-\frac{1}{\sqrt{(C V R \cdot C a \cdot H R \cdot 2 \pi)^{2}+1}}\right]
$$

\section{Association of eCPP With TAU}

The product of cerebrovascular resistance (CVR) and compliance $(\mathrm{Ca})$ represents the noninvasive time constant, or TAU. Therefore, the formula for eCPP (in $\mathrm{mm} \mathrm{Hg}$ ) (Eq. 2 in main text) can be expressed as:

$e C P P=A B P \cdot\left[0.734-\frac{0.266}{\sqrt{(T A U \cdot H R \cdot 2 \pi)^{2}+1}}\right]-7.026$

[Eq. C]

\section{References}

1. Aaslid R, Lindegaard KF, Sorteberg W, Nornes H: Cerebral autoregulation dynamics in humans. Stroke 20:45-52, 1989

2. Aaslid R, Lundar T, Lindegaard KF, Nornes H: Estimation of cerebral perfusion pressure from arterial blood pressure and transcranial Doppler recordings, in Miller JD, Teasdale GM, Rowan JO, et al (eds): Intracranial Pressure VI. New York: Springer-Verlag, 1986, pp 226-229, 1986

3. Allocca JA: Noninvasive monitoring of intracranial pressure. Med Electron 13:81-85, 1982

4. Andrews PJ, Sleeman DH, Statham PF, McQuatt A, Corruble $\mathrm{V}$, Jones PA, et al: Predicting recovery in patients suffering from traumatic brain injury by using admission variables and physiological data: a comparison between decision tree analysis and logistic regression. J Neurosurg 97:326-336, 2002

5. Belfort MA, Tooke-Miller C, Varner M, Saade G, Grunewald $\mathrm{C}$, Nisell H, et al: Evaluation of a noninvasive transcranial Doppler and blood pressure-based method for the assessment of cerebral perfusion pressure in pregnant women. Hypertens Pregnancy 19:331-340, 2000

6. Bouma GJ, Muizelaar JP: Cerebral blood flow, cerebral blood volume, and cerebrovascular reactivity after severe head injury. J Neurotrauma 9 (Suppl 1):S333-S348, 1992

7. Bratton SL, Chestnut RM, Ghajar J, McConnell Hammond FF, Harris OA, Hartl R, et al: Guidelines for the management of severe traumatic brain injury. IX. Cerebral perfusion thresholds. J Neurotrauma 24 Suppl 1:S59-S64, 2007 (Erratum in J Neurotrauma 25:276-278, 2008)

8. Budohoski KP, Schmidt B, Smielewski P, Kasprowicz M, Plontke R, Pickard JD, et al: Non-invasively estimated ICP pulse amplitude strongly correlates with outcome after TBI. Acta Neurochir Suppl 114:121-125, 2012

9. Bullock R, Chesnut RM, Clifton C, Ghajar J, Marion DW, Narayan RK, et al: Brain Trauma Foundation, American Association of Neurological Surgeons, Joint Section on Neurotrauma and Critical Care: Guidelines for the management of severe head injury. J Neurotrauma 13:641-734, 1996

10. Czosnyka M, Matta BF, Smielewski P, Kirkpatrick PJ, Pickard JD: Cerebral perfusion pressure in head-injured patients: a noninvasive assessment using transcranial Doppler ultrasonography. J Neurosurg 88:802-808, 1998

11. Czosnyka M, Richards H, Pickard JD, Harris N, Iyer V: Frequency-dependent properties of cerebral blood transport-an experimental study in anaesthetized rabbits. Ultrasound Med Biol 20:391-399, 1994
12. Czosnyka M, Smielewski P, Lavinio A, Czosnyka Z, Pickard JD: A synopsis of brain pressures: which? when? are they all useful? Neurol Res 29:672-679, 2007

13. Elf K, Nilsson P, Ronne-Engström E, Howells T, Enblad P: Cerebral perfusion pressure between 50 and $60 \mathrm{~mm} \mathrm{Hg}$ may be beneficial in head-injured patients: a computerized secondary insult monitoring study. Neurosurgery 56:962-971, 2005

14. Ghajar J: Traumatic brain injury. Lancet 356:923-929, 2000

15. Gobiet W, Grote W, Bock WJ: The relation between intracrainal pressure, mean arterial pressure and cerebral blood flow in patients with severe head injury. Acta Neurochir (Wien) 32:13-24, 1975

16. Hosmer DW, Lemeshow S: Applied Logistic Regression. New York: John Wiley \& Sons, 1989

17. Jones HA: Arterial transducer placement and cerebral perfusion pressure monitoring: a discussion. Nurs Crit Care 14:303-310, 2009

18. Kashif FM, Verghese GC, Novak V, Czosnyka M, Heldt T: Model-based noninvasive estimation of intracranial pressure from cerebral blood flow velocity and arterial pressure. Sci Transl Med 11:129ra44, 2012

19. Kasprowicz M, Czosnyka M, Soehle M, Smielewski P, Kirkpatrick PJ, Pickard JD, et al: Vasospasm shortens cerebral arterial time constant. Neurocrit Care 16:213-218, 2012

20. Kasprowicz M, Schmidt E, Kim DJ, Haubrich C, Czosnyka $\mathrm{Z}$, Smielewski P, et al: Evaluation of the cerebrovascular pressure reactivity index using non-invasive finapres arterial blood pressure. Physiol Meas 31:1217-1228, 2010</jrn>

21. Lassen NA: Control of cerebral circulation in health and disease. Circ Res 34:749-760, 1974

22. Maas AI, Dearden M, Teasdale GM, Braakman R, Cohadon F, Iannotti F, et al: EBIC-guidelines for management of severe head injury in adults. Acta Neurochir (Wien) 139:286294, 1997

23. Mayer SA, Chong JY: Critical care management of increased intracranial pressure. J Intensive Care Med 17:55-67, 2002

24. Mchedlishvili G: Physiological mechanisms controlling cerebral blood flow. Stroke 11:240-248, 1980

25. Michel E, Hillebrand S, vonTwickel J, Zernikow B, Jorch G: Frequency dependence of cerebrovascular impedance in preterm neonates: a different view on critical closing pressure. J Cereb Blood Flow Metab 17:1127-1131, 1997

26. Miller JD, Stanek A, Langfitt TW: Concepts of cerebral perfusion pressure and vascular compression during intracranial hypertension. Prog Brain Res 35:411-432, 1972

27. Narayan RK, Kishore PR, Becker DP, Ward JD, Enas GG, Greenberg RP, et al: Intracranial pressure: to monitor or not to monitor? A review of our experience with severe head injury. J Neurosurg 56:650-659, 1982

28. Nichol J, Girling F, Jerrard W, Claxton EB, Burton AC: Fundamental instability of the small blood vessels and critical closing pressures in vascular beds. Am J Physiol 164:330344,1951

29. Overgaard J, Tweed WA: Cerebral circulation after head injury. 1. Cerebral blood flow and its regulation after closed head injury with emphasis on clinical correlations. J Neurosurg 41:531-541, 1974

30. Panerai RB, Sammons EL, Smith SM, Rathbone WE, Bentley S, Potter JF, et al: Cerebral critical closing pressure estimation from Finapres and arterial blood pressure measurements in the aorta. Physiol Meas 27:1387-1402, 2006

31. Patel HC, Menon DK, Tebbs S, Hawker R, Hutchinson PJ, Kirkpatrick PJ: Specialist neurocritical care and outcome from head injury. Intensive Care Med 28:547-553, 2002

32. Puppo C, Camacho J, Yelicich B, Moraes L, Biestro A, Gomez $\mathrm{H}$ : Bedside study of cerebral critical closing pressure in patients with severe traumatic brain injury: a transcranial Doppler study. Acta Neurochir Suppl 114:283-288, 2012 
33. Rosner MJ: Role of cerebral perfusion pressure in acute brain trauma. Crit Care Med 24:1274-1276, 1996 (Letter)

34. Rosner MJ, Daughton S: Cerebral perfusion pressure management in head injury. J Trauma 30:933-941, 1990

35. Rosner MJ, Rosner SD, Johnson AH: Cerebral perfusion pressure: management protocol and clinical results. J Neurosurg 83:949-962, 1995

36. Sammons EL, Samani NJ, Smith SM, Rathbone WE, Bentley S, Potter JF, et al: Influence of noninvasive peripheral arterial blood pressure measurements on assessment of dynamic cerebral autoregulation. J Appl Physiol (1985) 103:369-375, 2007

37. Schmidt EA, Czosnyka M, Gooskens I, Piechnik SK, Matta $\mathrm{BF}$, Whitfield PC, et al: Preliminary experience of the estimation of cerebral perfusion pressure using transcranial Doppler ultrasonography. J Neurol Neurosurg Psychiatry 70:198-204, 2001

38. Schmidt EA, Czosnyka M, Matta BF, Gooskens I, Piechnik S, Pickard JD: Non-invasive cerebral perfusion pressure (nCPP): evaluation of the monitoring methodology in head injured patients. Acta Neurochir Suppl 76:451-452, 2000

39. Steiner LA, Andrews PJ: Monitoring the injured brain: ICP and CBF. Br J Anaesth 97:26-38, 2006

40. Varsos GV, Richards H, Kasprowicz M, Budohoski KP, Brady KM, Reinhard M, et al: Critical closing pressure deter- mined with a model of cerebrovascular impedance. J Cereb Blood Flow Metab 33:235-243, 2013

41. Vespa P: What is the optimal threshold for cerebral perfusion pressure following traumatic brain injury? Neurosurg Focus 15(6):E4, 2003

\section{Author Contributions}

Conception and design: GV Varsos, Czosnyka. Acquisition of data: Czosnyka. Analysis and interpretation of data: GV Varsos, Brady, Hutchinson, Pickard, Czosnyka. Critically revising the article: Kolias, Smielewski, Brady, VG Varsos, Hutchinson, Pickard, Czosnyka. Reviewed submitted version of manuscript: Czosnyka. Approved the final version of the manuscript on behalf of all authors: GV Varsos. Statistical analysis: GV Varsos. Administrative/technical/material support: Czosnyka. Study supervision: Czosnyka.

\section{Correspondence}

Georgios V. Varsos, Division of Neurosurgery, Department of Clinical Neurosciences, Addenbrooke's Hospital, University of Cambridge, Hills Rd., Cambridge CB2 0QQ, United Kingdom. email: gv249@cam.ac.uk. 